\title{
Inhaler technique in patients attending an urban pulmonology practice
}

\author{
J Vanderwagen, Registered Nurse and Midwife; C Smith, MB BCh, FCP (SA), MMed (Int), FCCP \\ Morningside Mediclinic, Johannesburg, South Africa
}

Corresponding author: J Vanderwagen (csmithtrials@mweb.co.za)

\begin{abstract}
Objective. To evaluate the use of inhaler therapy, primarily focusing on the source of the initial inhaler training and the effects of regular monitoring of inhaler use.

Methods. We conducted a prospective study of 200 adult patients using either a metered dose inhaler (MDI) or dry powder inhaler (DPI), attending a private pulmonology practice for the treatment of asthma, chronic obstructive pulmonary disease (COPD) or asthma-COPD overlap. Each patient was evaluated once, and assessed by the investigators irrespective of the patient's background and inhaler technique training.

Results. The MDI and DPI techniques were found to be $45 \%$ and $79 \%$ adequate, respectively $(p<0.001)$. Patients who had initial training in a specialist or pulmonology practice showed $100 \%$ adequacy. In stark contrast, inadequate technique was observed where initial MDI training was performed by family members, general practitioners, hospital staff, pharmacy staff and self $(p<0.05)$. Similarly, inadequate technique was seen in the DPI group when taught by family members, general practitioners, hospital staff and pharmacy staff $(p<0.001)$. The Accuhaler was used by $59 \%$ of the patients using a DPI; $41 \%$ used the Turbuhaler. The percentage of inadequate inhaler technique in the Accuhaler was $28 \%$, while the percentage of inadequate inhaler use in the Turbuhaler group was lower at $10 \%(p<0.001)$.

Conclusion. In an urban private practice environment, the adequacy of inhaler technique was shown to be suboptimal. DPI technique was found to be superior to MDI technique, and the Turbuhaler was employed more adequately than the Accuhaler. There was no correlation between the duration of inhaler use and technique, but patients initially taught by non-pulmonology specialists and pulmonologists showed superior technique to that of other groups, i.e. patients taught by family members, general practitioners and hospital staff.
\end{abstract}

S Afr Respir J 2017;23(1):5-7. DOI:10.7196/SARJ.2017.v23i1.97

It is well known that difficulty in the use of inhalers may negatively impact their subsequent benefit. Control of lung disease is essential, as it has a significant effect on the number of admissions and exacerbations, which leads to higher healthcare costs. ${ }^{[1]}$

Numerous devices have been marketed with adaptations to suit different patient preferences, but the proper and regular training of patients when the device is prescribed is lacking. ${ }^{[2]}$ An inhaled corticosteroid is the drug of choice in the treatment of patients with persistent asthma, ${ }^{[3]}$ and successful delivery of the drug is crucial.

The aim of this study was to prospectively evaluate the use of inhaler therapy, primarily focusing on the source of the initial inhaler training and the effects of regular monitoring of inhaler use.

\section{Methods}

We conducted a prospective study on 200 adult patients over a 6-month period from August 2015 to January 2016, using either a metered dose inhaler (MDI) or dry powder inhaler (DPI) in a private pulmonology practice, for the treatment of asthma, chronic obstructive pulmonary disease (COPD) or asthma-COPD overlap syndrome (ACOS).

The data apply to a single evaluation episode per patient, which assessed: (i) the device used; (ii) inhaler technique; and (iii) who the patient was initially trained by.
Adequacy of inhaler technique was determined according to the manufacturer's recommendations using placebo devices. For MDI, the patients were required to shake the inhaler before use, remove the cap, breathe out away from the inhaler, put the inhaler in the mouth and press the inhaler while taking a slow, deep breath in, and hold their breath for $\sim 10$ seconds after removing the MDI from their mouth. For Accuhaler technique, the patients were required to open the Accuhaler correctly for exposure of the mouthpiece, press the lever down until it stopped, exhale (completely) away from the mouthpiece, place lips around the mouthpiece and breathe in steadily through the Accuhaler, remove from lips and hold breath for $\sim 10$ seconds afterwards. For Turbuhaler technique, the patients were required to unscrew and remove the cap. While holding the Turbuhaler upright, they should have twisted the coloured grip as far as it would go and then twisted it all the way back again until a click was heard, exhaled totally away from the mouthpiece, put the mouthpiece between their teeth, closed lips around and breathe in forcefully and deeply, and then remove the Turbuhaler from their mouth before breathing out. Part of the inhaler technique assessment was ensuring that the patients using inhaled corticosteroids rinsed their mouth after use of their device.

This information was analysed to compare MDI and DPI techniques, to assess whether the techniques were adequate or inadequate according to the guidelines given by the pharmaceutical company, 
and to evaluate whether the duration of therapy was a marker for good or poor technique.

The statistical technique used was to test the hypothesis of the difference of proportions in two independent populations. The null hypothesis was that the proportions were equal in both populations, whereas the alternative hypothesis was chosen appropriately for each case. Appropriate use of $z$-statistics and Student $t$-statistics was allowed for as well.

\section{Results}

\section{MDI v. DPI}

On comparing the overall inhaler technique of the MDI v. the DPI, the use of the MDI was found to be inferior. Of the patients, 179 were using DPIs and 175 were using the MDI. A total of 154 patients were using both devices. The MDI and DPI techniques were found to be $45 \%$ and $79 \%$ adequate, respectively $(p<0.001)$. Inadequate technique was associated with poor co-ordination and incorrect use of the device (Fig. 1). Information about prior device usage and switching from one device to another because of difficulty in using a particular inhaler was not obtained during this study.

\section{Initial training given}

Seven areas of initial training were compared for both the MDI and DPI. A marked inadequacy of inhaler technique was observed in patients whose initial MDI training was performed by family members $(n=19)$, general practitioners $(n=49)$, hospital staff, i.e. nursing staff dispensing medication $(n=9)$, pharmacy staff $(n=20)$ and self $(n=4) \quad(p<0.05)$ (Fig. 2). Similarly, inadequate technique was seen in the DPI group when taught by family members $(n=7)$, general practitioners $(n=17)$, hospital staff $(n=5)$ and pharmacy staff $(n=14)(p<0.001)$ (Fig. 3$)$. The study revealed that initial training of both MDI (73 patients) and DPI (132 patients) in a non-pulmonology or pulmonology practice showed $100 \%$ adequacy. This may be related to the time available for training of the patients or the availability of suitably trained staff.

\section{Duration of use}

There was no correlation between adequacy of the MDI or DPI and the duration of use of the inhaler, whether used for $<1$ year or $>10$ years, as seen in Figs 4 and 5.

\section{Accuhaler v. Turbuhaler}

With respect to DPI use, we collected a record of the patients using the Accuhaler (104 patients) and Turbuhaler (71 patients). In a study

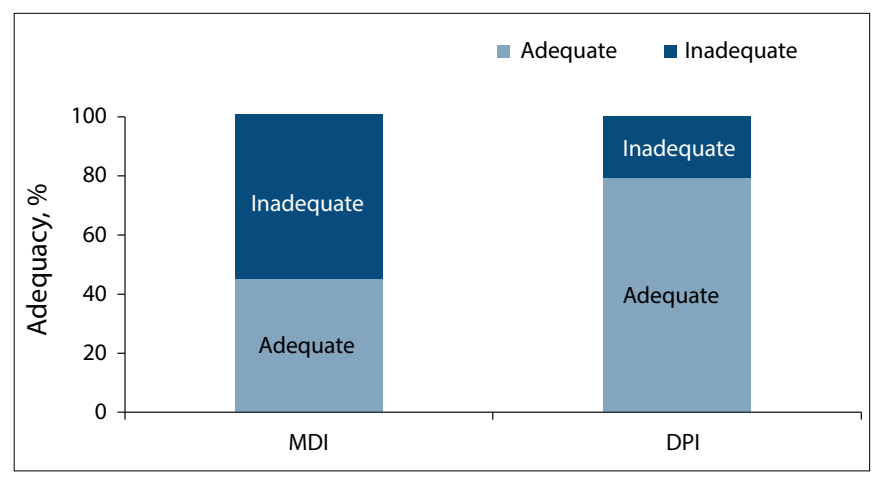

Fig. 1. Adequacy of MDI and DPI technique $(\mathrm{p}<0.001)$.

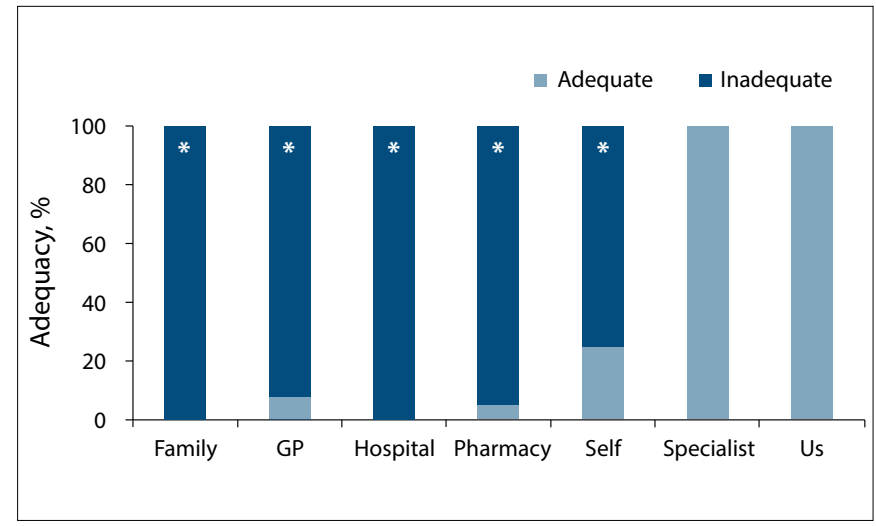

Fig. 2. MDI technique and initial training given. $\left({ }^{*} \mathrm{p}<0.05\right)$

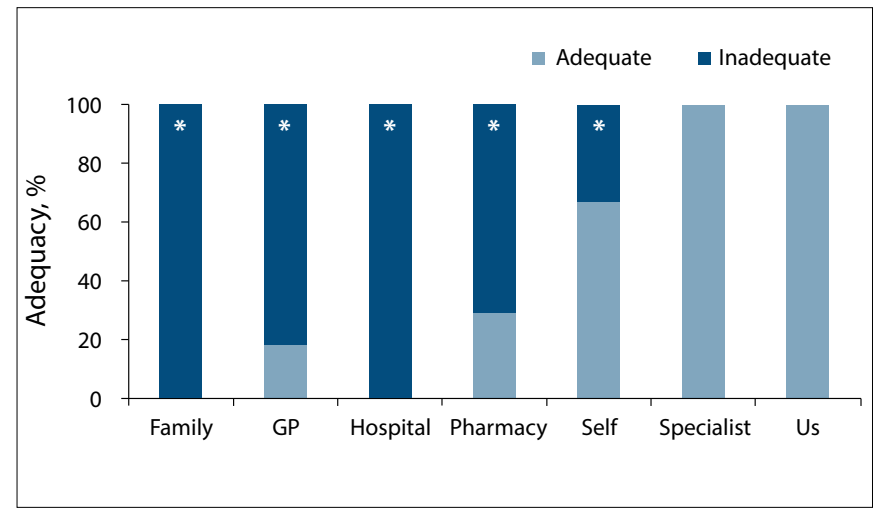

Fig. 3. DPI technique and initial training given. $\left({ }^{*} \mathrm{p}<0.001\right)$

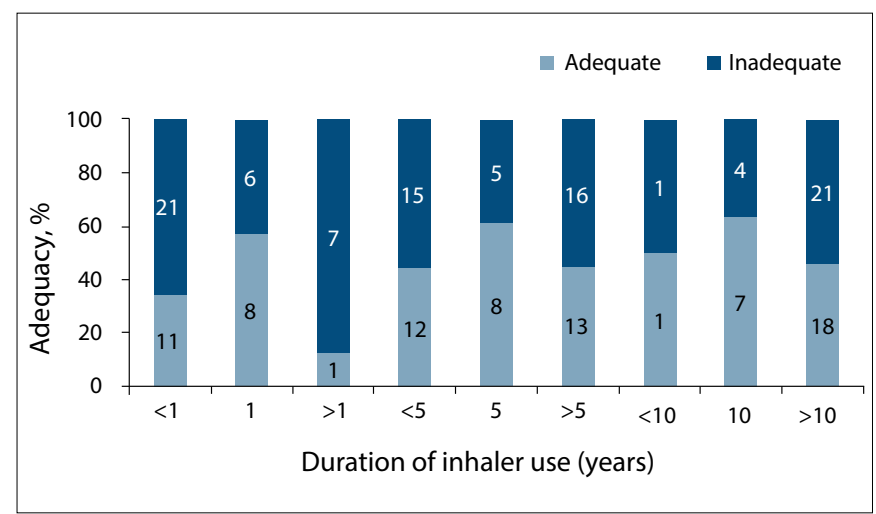

Fig. 4. MDI technique and duration of use.

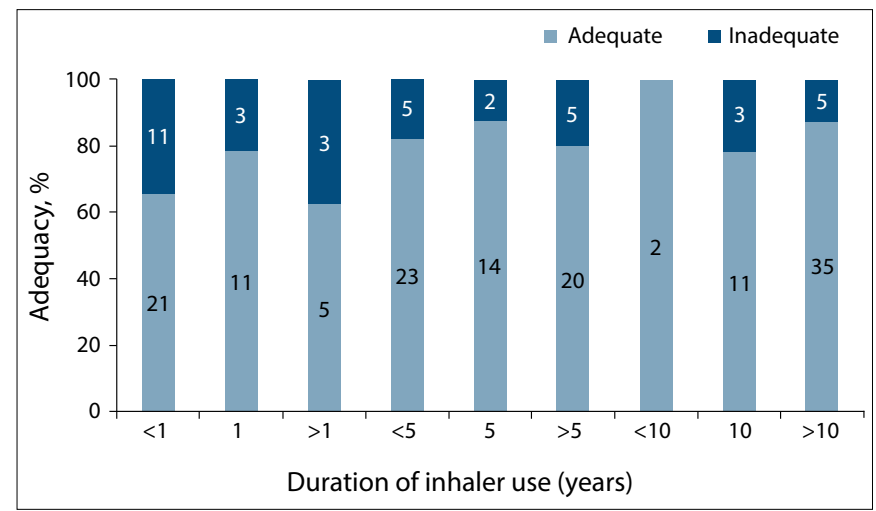

Fig. 5. DPI technique and duration of use. 


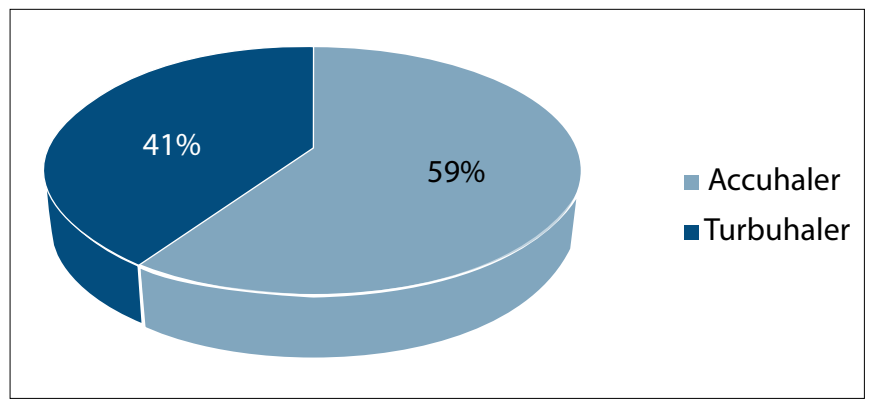

Fig. 6. DPI percentage use comparing the Accuhaler and Turbuhaler.

conducted in 2008, it was found that the Accuhaler was associated with a lower rate of incorrect handling than the Turbuhaler. ${ }^{[4]}$ We included a sub-study comparing the Accuhaler and Turbuhaler, looking at percentage use between the two devices (Fig. 6) and technique inadequacies for both the DPI devices (Figs 7 and 8), in order to determine if this was still the case.

Of the patients using a DPI, 59\% used the Accuhaler and $41 \%$ used the Turbuhaler. Although the percentage of Accuhaler users was considerably higher, the percentage of inadequate inhaler technique in the Accuhaler group was $28 \%$, while the percentage of inadequate inhaler use in the Turbuhaler group was lower at $10 \%(p<0.001)$.

\section{Discussion}

In this urban-based, pulmonologist private practice population, we documented overall suboptimal inhaler technique use. However, DPI devices fared better than MDI devices. The reasons for this are uncertain, as the assumption would be that the same level of training was given to all patients. A possible explanation may be that patients are able to better train themselves using the DPI device, as a similar proportion used DPIs $(n=179)$ and MDIs $(n=175)$. There was no correlation between the duration of inhaler use and the technique used for the inhaler. Patients trained in a specialist and pulmonology practice showed superior technique to other groups in the study. This may simply relate to adequate time provided for training, together with teaching provided by staff educated in this area. In comparing two DPI devices, the Turbuhaler and Accuhaler, it was found that the Accuhaler was more widely used (68\% Accuhaler v. 32\% Turbuhaler). The patients' Turbuhaler technique (90\% adequate) was superior to Accuhaler technique (72\% adequate). The reasons for the superiority of the use of the Turbuhaler are not clear and cannot be ascertained from the study.

However, Sanchis et al. ${ }^{[5]}$ reinforced the fact that no drug is effective until delivery to the site of action, and regular inhaler technique monitoring and training need to be performed, irrespective of the device type used or how long the patient has been using the medication.

The most likely reason for inhaler technique being so poor relates to inadequate training. It is suspected that the teachers themselves, including non-specialist doctors, nursing staff and pharmacists, do not know the correct technique for using different inhaler devices. Another reason could relate to the time available to doctors, nursing

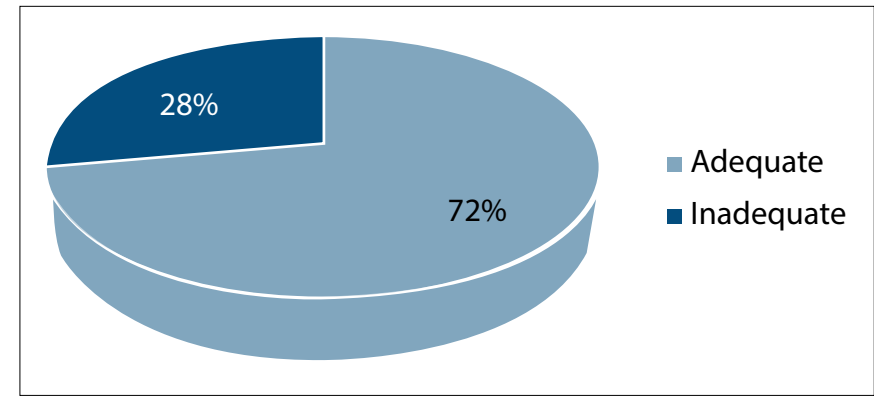

Fig. 7. Adequacy of the Accuhaler technique ( $<<0.001)$.

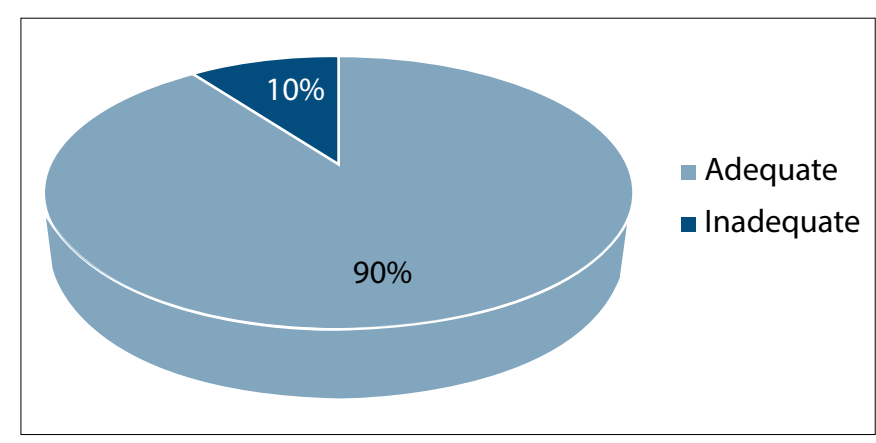

Fig. 8. Adequacy of the Turbuhaler technique ( $\mathrm{p}<0.001)$.

staff and pharmacists to check the patient's inhaler technique and to provide necessary corrective measures.

The situation may be improved by incorporating training on the use of the inhaler devices as a part of continuing medical education for medical, pharmacy and nursing students, as well as doctors. The importance of regular monitoring of inhaler technique must be emphasised at all levels of healthcare. We would encourage the manufacturers of these devices to champion the drive to make sure their inhaler devices are used appropriately across all spheres of medicine.

\section{Conclusion}

Suboptimal use of inhalers for the management of obstructive lung disease remains a serious obstacle to the adequate management of these patients. This study highlights a significant opportunity for improving the control of obstructive lung disease by utilising improved training methods at the prescription level.

1. Van Blydenstein SA, Nqwata L, Banda NPK, Ashmore P, Wong ML. Factors affecting compliance and control of asthma in patients attending the Respiratory Outpatient Department, Chris Hani Baragwanath Academic Hospital. S Afr Respir J 2015;21(4):9195. https://doi.org/10.7196/sarj.2015.v21i4.43

2. Mash B, Rhode H, Pather M, et al. Quality of asthma care: Western Cape Province, South Africa. S Afr Med J 2009;99(12):892-896

3. Fanta CH. Asthma. New Engl J Med 2009;360(10):1002-1014. https://doi.org/10.1056/ nejmra0804579

4. Khassawneh BY, Al-Ali MK, Alzoubi KH, et al. Handling of inhaler devices in actual pulmonary practice: Metered-dose inhaler versus dry powder inhalers. Respir Care 2008;53(3):324-328.

5. Sanchis J, Corrigan C, Levy ML, Viejo JL. Inhaler devices - from theory to practice. Respir Med 2013;107(4):495-502. https://doi.org/10.1016/j.rmed.2012.12.007 